\section{Unfairness for mouth cancer patients}

We wish to draw to your attention the unfair situation faced by many mouth cancer patients compared to most other forms of cancers.

It is an issue that needs to be put right in any new dental commissioning arrangements to ensure that mouth cancer sufferers do not have the financial burden for recovering from their condition and returning their lives to normal.

Macmillan Cancer Support have recently highlighted in the media the financial burden affecting cancer patients, with two thirds (66\%) reporting an increase in costs are a result of suffering from cancer. For many mouth cancer sufferers the financial challenges are even more daunting. During Mouth Cancer Action Month we believe it is important to raise the financial inequalities that some patients with mouth cancer have to endure in addition to the burden of their disease.

Within the hospital system mouth cancer patients receive all their treatment free of charge in line with patients with other cancers. Sadly, this is not the case outside the hospital setting. The nature of treatment for mouth cancer, irrespective of the modality, has a significant impact upon the teeth as well as the soft tissues of the mouth as a direct consequence of treatment. This often leaves the patient with significant lifelong dental needs. If they were fortunate to have access to a restorative specialist, who was linked to their cancer unit, this treatment would be free.

However, where these resources are unavailable, or where there are long waiting times, patients have to register with their local dentist and pay for routine treatment. Some may view full mouth rehabilitation following surgery with implants, bridges and crowns as non-essential aesthetic treatment, only available privately. However, giving a patient who has had major facial surgery their smile back is often a major priority for the patient. In our opinion there would be a public outcry if patients with any other type cancer were asked to contribute to the cost of their reconstructive treatment.

Given the uncertainties over the new dental contract and the commissioning of future dental services we are concerned that patients who have treatment for mouth cancer, and who cannot have all their follow up in the hospital setting, will continue to be forced to pay to maintain their oral health.

We think it is time for the dental profession to stand up for patients and we have established an e-petition to encourage government to add patients who have had treatment for mouth cancer to be exempt from NHS dental charges. We hope all dental professionals will support the e-petition which can be signed by visiting: http://epetitions. direct.gov.uk/petitions/22063.

We also feel strongly about the limited availability of free examinations for mouth cancer. Improvements in survival rates for mouth cancer are lagging behind most other cancers. Early detection is vital and without changes to the current arrangements 30,000 people are likely to lose their life to mouth cancer over the next decade. These figures are greater than cervical and testicular cancer combined.

We believe that all patients should have a right to access free professional care when they are worried about having cancer. A patient with a breast lump or a testicular swelling can see their general medical practitioner to be examined and referred for free whereas a patient with a mouth ulcer who is worried that a mouth ulcer may be cancerous will have to pay for that same privilege unless they fit into one of the NHS exemption categories.

Often dental patients who cannot get access to an NHS dentist are forced to pay significant amounts of money for this service through the private route. Furthermore those patients who are unable to afford the price of a private consultation may see their doctor for a free consultation. Sadly there are still too many cases where malignancies are missed or poorly managed due to the lack of training of medical professionals in diagnosing mouth cancer.

Although the cost of an NHS check-up, which is meant to include an examination of the oral mucosa, may not seem like a big expense it may be a significant cost to those on low incomes in the current economic climate. Furthermore given that a low socio-economic status is an independent risk factor for mouth cancer this may dis-incentivise the very people who are at a higher risk of developing mouth cancer from getting timely professional advice.

If we want to really make an impact on the statistics and support for mouth cancer sufferers in the UK we cannot ignore the unfairness of the current arrangements. We hope it is an issue that the whole profession will support over the coming months.

\section{Trivedy \\ N. Carter}

This letter was first published in the $\mathrm{BDJ}$ on 14 January 2012 (Volume 212 page 3). 